\title{
Low-rank retractions: a survey and new results*
}

\author{
P.-A. Absil ${ }^{\dagger} \quad$ I. V. Oseledets ${ }^{\ddagger}$
}

November 4, 2014

\begin{abstract}
Retractions are a prevalent tool in Riemannian optimization that provides a way to smoothly select a curve on a manifold with given initial position and velocity. We review and propose several retractions on the manifold $\mathcal{M}_{r}$ of rank- $r m \times$ matrices. With the exception of the exponential retraction (for the embedded geometry), which is clearly the least efficient choice, the retractions considered do not differ much in terms of run time and flop count. However, considerable differences are observed according to properties such as domain of definition, boundedness, first/second-order property, and symmetry.
\end{abstract}

Key words: Low-rank manifold; fixed-rank manifold; low-rank optimization; retraction; geodesic; quasi-geodesic; projective retraction; orthographic retraction; Lie-Trotter splitting

\section{Introduction}

We consider the general low-rank optimization problem of minimizing a real-valued function on a set of matrices of fixed rank:

$$
\min _{X \in \mathcal{M}_{r}} f(X)
$$

where

$$
\mathcal{M}_{r}=\left\{X \in \mathbb{R}^{m \times n}: \operatorname{rank}(X)=r\right\}
$$

is the set of $m \times n$ matrices of rank $r$ and $m, n$, and $r<\min (m, n)$ are positive integers. Applications of (11) appear in particular in learning problems, where the low-rank constraint is inherent to the model or introduced to reduce memory usage and computation time; see the list of applications in the introduction of [MMBS13b].

Several techniques have been proposed to address (11) - or more specific instances thereof - by exploiting the fact that $\mathcal{M}_{r}$ is a submanifold of the Euclidean space $\mathbb{R}^{m \times n}$; see, e.g., MMBS13a,

\footnotetext{
*This paper presents research results of the Belgian Network DYSCO (Dynamical Systems, Control, and Optimization), funded by the Interuniversity Attraction Poles Programme initiated by the Belgian Science Policy Office. This work was financially supported by the Belgian FRFC (Fonds de la Recherche Fondamentale Collective). The work of I.O. was supported by Russian Science Foundation grant 14-11-00659. The final publication is available at Springer via http://dx.doi.org/10.1007/s10589-014-9714-4

${ }^{\dagger}$ Department of Mathematical Engineering, ICTEAM Institute, Université catholique de Louvain, B-1348 Louvain-la-Neuve, Belgium (http://sites.uclouvain.be/absil/).

${ }^{\ddagger}$ Institute of Numerical Mathematics, Russian Academy of Sciences, Gubkina Street 8, Moscow, Russia (ivan.oseledets@gmail.com).
} 
SWC13, AAM14, MMBS13b, Van13. Most of these techniques choose a descent direction $\dot{X}$ for $f$ in the tangent space to $\mathcal{M}_{r}$ at the current iterate $X \in \mathcal{M}_{r}$ and then compute the next iterate by performing a line search along a curve $\gamma$ on $\mathcal{M}_{r}$ satisfying $\gamma(0)=X$ and $\left.\frac{\mathrm{d}}{\mathrm{d} t} \gamma(t)\right|_{t=0}=\dot{X}$. The curve $\gamma$ is conveniently chosen as $\gamma(t)=R(X, t \dot{X})$ where $R$ is a retraction on $\mathcal{M}_{r}$. Retractions on manifolds, a concept due to Shub [Shu86] that we recall in Section 2.3, have received much attention lately in the context of Riemannian optimization; see, e.g., $\mathrm{ADM}^{+} 02$, $\mathrm{ABG07}, \mathrm{AMS08}$ Van13, AM12, RW12, SWC13, BMAS14, KSV14.

The purpose of this paper is to review several retractions on $\mathcal{M}_{r}$ and propose new ones. In particular, we introduce the Lie-Trotter retraction, which directly follows from the first-order splitting method described in [LO13, §3.2]. An extended version of the "KSL" flavor of this retraction is known to have an exactness property [LO13, Theorem 4.1], from which we deduce that the Lie-Trotter retraction is a second-order retraction, i.e., the second derivative of $t \mapsto R(X, t \dot{X})$ at $t=0$ belongs to the normal space to $\mathcal{M}_{r}$ at $X$.

The paper is organized as follows. After Section 2 giving the necessary background and preliminaries, Section 3 presents the various retractions, discussing their implementation and their computational cost. Numerical experiments comparing the retractions are conducted in Sections 4 and 5. Conclusions are drawn in Section 6.

\section{Background and preliminaries}

This section recalls fundamental notions pertaining to the low-rank manifolds, flop counts, and retractions on manifolds.

\subsection{The low-rank manifold}

Central in this paper is the low-rank manifold $\mathcal{M}_{r}$ (2). This subsection gives background on the geometry of $\mathcal{M}_{r}$, with an emphasis on the representation of its elements and tangent vectors.

We first introduce some notation. Let

$$
\operatorname{St}(r, m)=\left\{X \in \mathbb{R}^{m \times r}: X^{\top} X=I_{r}\right\}
$$

denote the (compact) Stiefel manifold of orthonormal $m \times r$ matrices,

$$
\mathbb{R}_{*}^{m \times r}=\left\{X \in \mathbb{R}^{m \times r}: \operatorname{rank}(X)=r\right\}
$$

denote the noncompact Stiefel manifold of full column rank $m \times r$ matrices,

$$
\mathrm{GL}(r)=\left\{X \in \mathbb{R}^{r \times r}: \operatorname{rank}(X)=r\right\}
$$

denote the general linear group of order $r$, i.e., the set of all $r \times r$ invertible matrices, and

$$
\mathrm{O}(r)=\left\{X \in \mathbb{R}^{r \times r}: X^{\top} X=I_{r}\right\}
$$

denote the orthogonal group of order $r$, i.e., the set of all $r \times r$ orthogonal matrices. 
The set $\mathcal{M}_{r}$ is known to be a submanifold of dimension $(m+n-r) r$ embedded in the Euclidean space $\mathbb{R}^{m \times n}$ [Lee03, Example 8.14]. The low-rank optimization problem (11) is thus in the field of play of Riemannian optimization; see, e.g., AMS08.

In practice, we prefer not to store an $X \in \mathcal{M}_{r}$ as an $m \times n$ matrix; it requires storing $m n$ numbers, which is much larger than the manifold dimension $(m+n-r) r$ in the frequent situation where $r \ll \min (m, n)$. Instead, we represent $X \in \mathcal{M}_{r}$ as

$$
\begin{aligned}
& X=M N^{\top} \text { with }(M, N) \in \mathcal{N}_{1}:=\mathbb{R}_{*}^{m \times r} \times \mathbb{R}_{*}^{n \times r}, \text { or } \\
& X=M N^{\top} \text { with }(M, N) \in \mathcal{N}_{2}:=\operatorname{St}(r, m) \times \mathbb{R}_{*}^{n \times r}, \text { or } \\
& X=U S V^{\top} \text { with }(U, S, V) \in \mathcal{N}:=\operatorname{St}(r, m) \times \operatorname{GL}(r) \times \operatorname{St}(r, n) .
\end{aligned}
$$

Several other representations exist, see [MMBS13b, §3], but in this paper we will only make use of the three representations above, with an emphasis on (5). Note that the mappings

$$
\pi_{i}: \mathcal{N}_{i} \rightarrow \mathcal{M}_{r}:(M, N) \mapsto M N^{\top}
$$

$i=1,2$, and

$$
\pi: \mathcal{N} \rightarrow \mathcal{M}_{r}:(U, S, V) \mapsto U S V^{\top}
$$

are surjective (i.e., every $X \in \mathcal{M}_{r}$ is represented) but not injective: the equivalence classes of representations are

$$
\begin{aligned}
& \pi_{1}^{-1}\left(\pi_{1}(M, N)\right)=\left\{\left(M R, N R^{-T}\right): R \in \mathrm{GL}(r)\right\} \\
& \pi_{2}^{-1}\left(\pi_{2}(M, N)\right)=\{(M Q, N Q): Q \in \mathrm{O}(r)\} \\
& \pi^{-1}(\pi(U, S, V))=\left\{\left(U Q_{U}, Q_{U}^{\top} S Q_{V}, V Q_{V}\right): Q_{U}, Q_{V} \in \mathrm{O}(r)\right\} .
\end{aligned}
$$

We also point out, as we will allude to this fact later on, that each of the three " $\pi$ " mappings is a submersion, i.e., its differential is surjective at every point; this is shown in detail in AAM14, $\S 2]$ for the case of $\pi_{1}$, and the two other cases can be treated similarly. This provides us with three different expressions of $\mathcal{M}_{r}$ as a quotient manifold. The one that concerns us most is

$$
\mathcal{M}_{r} \simeq(\mathrm{St}(r, m) \times \mathrm{GL}(r) \times \mathrm{St}(r, n)) /(\mathrm{O}(r) \times \mathrm{O}(r))
$$

with quotient map (6) whose fibers are given by (7).

The set of all tangent vectors to $\mathcal{M}_{r}$ at $X=U S V^{\top}$ (5) is termed the tangent space to $\mathcal{M}_{r}$ at $X$ and denoted by $\mathrm{T}_{X} \mathcal{M}_{r}$. The concept of tangent vector to an abstract manifold can be found, e.g., in Boo03 or AMS08. Since $\mathcal{M}_{r}$ is a submanifold of $\mathbb{R}^{m \times n}$, the tangent space $\mathrm{T}_{X} \mathcal{M}_{r}$ is simply identified with $\left\{\gamma^{\prime}(0): \gamma\right.$ smooth curve on $\mathcal{M}_{r}$ with $\left.\gamma(0)=X\right\}$. Depending of whether we want to recall the foot $X$ in the notation, we write $(X, \dot{X}) \in \mathrm{T}_{X} \mathcal{M}_{r}$ or $\dot{X} \in \mathrm{T}_{X} \mathcal{M}_{r}$.

The projection $\mathcal{P}_{X} Z$ of $Z \in \mathbb{R}^{m \times n}$ onto the tangent space $\mathrm{T}_{X} \mathcal{M}_{r}$ is given by

$$
\mathcal{P}_{X} Z=Z V V^{\top}+U U^{\top} Z-U U^{\top} Z V V^{\top}
$$

see [KL07, Lemma 4.1]. Hence every tangent vector $\dot{X}$ to $\mathcal{M}_{r}$ at $X \in \mathcal{M}_{r}$ can be written in the form

$$
\dot{X}=Z V V^{\top}+U U^{\top} Z-U U^{\top} Z V V^{\top}
$$


However, the choice of $Z$ to represent $\dot{X}$ is not unique, and moreover $Z \in \mathbb{R}^{m \times n}$ contains again $m n$ numbers, to be compared with the dimension $(m+n-r) r$ of the vector space $\mathrm{T}_{X} \mathcal{M}_{r}$. These drawbacks are remedied next.

Once a decomposition (5) is chosen for $X \in \mathcal{M}_{r}$, a unique representation $(\dot{U}, \dot{S}, \dot{V})$ of any $\dot{X} \in \mathrm{T}_{X} \mathcal{M}_{r}$ can be chosen such that

$$
\begin{gathered}
\dot{X}=U \dot{S} V^{\top}+\dot{U} S V^{\top}+U S \dot{V}^{\top}, \\
U^{\top} \dot{U}=0, \quad V^{\top} \dot{V}=0 .
\end{gathered}
$$

This follows from [KL07, §2.1], or alternatively from the machinery of quotient manifolds by showing that $(U, S, V) \mapsto\left\{(\dot{U}, \dot{S}, \dot{V}): U^{\top} \dot{U}=0, V^{\top} \dot{V}=0\right\}$ is a horizontal distribution for the quotient (8). If $\dot{X}=\mathcal{P}_{X} Z$, and in particular if $\dot{X}=Z$, the decomposition (11) is given by

$$
\begin{aligned}
\dot{S} & =U^{\top} Z V, \\
\dot{U} & =(Z V-U \dot{S}) S^{-1}=\left(I-U U^{\top}\right) Z V S^{-1}, \\
\dot{V} & =\left(Z^{\top} U-V \dot{S}^{\top}\right) S^{-T}=\left(I-V V^{\top}\right) Z^{\top} U S^{-T} .
\end{aligned}
$$

In order to get rid of the inverse of $S$ that appears in the above formulas, we can set $U_{p}:=\dot{U} S$ and $V_{p}=\dot{V} S$, which yields the unique representation considered in Van13, §2.1]:

$$
\begin{gathered}
\dot{X}=U \dot{S} V^{\top}+U_{p} V^{\top}+U V_{p}^{\top}, \\
U^{\top} U_{p}=0, V^{\top} V_{p}=0 .
\end{gathered}
$$

We will favor this representation. If $\dot{X}=\mathcal{P}_{X} Z$, and in particular if $\dot{X}=Z$, the decomposition (12) is given by

$$
\begin{aligned}
\dot{S} & =U^{\top} Z V \\
U_{p} & =Z V-U \dot{S}=\left(I-U U^{\top}\right) Z V \\
V_{p} & =Z^{\top} U-V \dot{S}^{\top}=\left(I-V V^{\top}\right) Z^{\top} U .
\end{aligned}
$$

Finally, the normal space at $X$ is the orthogonal complement to $\mathrm{T}_{X} \mathcal{M}_{r}$, in the sense of the classical Frobenius inner product in the embedding space $\mathbb{R}^{m \times n}$. Since the tangent space is given by

$$
\mathrm{T}_{X} \mathcal{M}_{r}=\left\{U \dot{S} V^{\top}+U_{p} V^{\top}+U V_{p}^{\top}: \dot{S} \in \mathbb{R}^{r \times r}, U_{p} \in \mathbb{R}^{m \times r}, U^{\top} U_{p}=0, V_{p} \in \mathbb{R}^{n \times k}, V^{\top} V_{p}=0\right\}
$$

one finds that the normal space is

$$
T_{X}^{\perp} \mathcal{M}_{r}=\left\{Z \in \mathbb{R}^{m \times n}: U^{\top} Z=0 \text { and } Z V=0\right\} .
$$

\subsection{Flop counts}

We will use flop counts as a way to compare the computational cost of various operations. When counting flops, we follow the conventions in [GV96, §1.2.4], and we always assume, unless otherwise stated, that $m<n$, as this has an impact on the way certain computations are carried out. For 
example, $U^{\top} Z V$ costs $2 m n r+2 m r^{2}$ flops when performed as $U^{\top}(Z V)$ and $2 m n r+2 n r^{2}$ flops when performed as $\left(U^{\top} Z\right) V$; the assumption that $m<n$ will thus lead us to prefer the $U^{\top}(Z V)$ order, unless $U^{\top} Z$ is needed in later operations and $Z V$ not, in which case the $\left(U^{\top} Z\right) V$ order is preferable. These are subtle considerations that need to be taken into account when looking for the most flop-efficient implementation. This calls for two caveats. The first one is that we have made a careful but not systematic attempt to find the most flop-efficient implementation of the various retractions studied in this paper.

The second caveat, much in line with the last paragraph of [GV96, §1.2.4], is that the flop count does not directly translate into time efficiency, as it ignores various overheads - such as memory traffic - associated with program execution. For example, if $A$ and $B$ are $m \times r$ and $C$ is $r \times r$ with $m=10^{4}$ and $r=10^{2}$, then computing $\mathrm{A}^{\prime} * \mathrm{~B}$ in Matlab can be significantly slower than computing $\mathrm{A} * \mathrm{C}$, even though both operations have the same the flop count of $2 m r^{2}$. Moreover, even though it can also be performed in $2 m r^{2}$ flops with modified Gram-Schmidt [GV96, §5.2.8], the economy-size QR decomposition of $A$ in Matlab is slower than the product $\mathrm{A} * \mathrm{C}$, even much so on older versions of Matlab. In order to avoid relying on Matlab's QR implementation, we perform orthonormalizations using the polar decomposition $A=Q P$ with $P=\left(A^{\top} A\right)^{1 / 2}$ and $Q=A / P$, for a dominant flop count of $4 m r^{2}$ when $A$ is of size $m \times r$ with $r \ll m$.

These remarks indicate that the flop counts and the timing comparisons given in this paper, while informative, must be taken with a grain of salt. With these provisions in mind, we obtain that computing $\dot{X}=\mathcal{P}_{X} Z$ (9) in the $\left(U_{p}, \dot{S}, V_{p}\right)$ representation (12), with $X$ given in the $(U, S, V)$ representation (5), requires a flop count of $2 m n r[Z V]+2 m r^{2}\left[U^{\top} Z V\right]+2 m r^{2}[U \dot{S}]+2 m n r\left[Z^{\top} U\right]+$ $2 n r^{2}\left[V \dot{S}^{\top}\right]$, hence $4 m n r+4 m r^{2}+2 n r^{2}$.

Throughout the paper, we present dominant flop counts under the common assumption that the rank is very low, i.e., $r \ll m$. For $\mathcal{P}_{X} Z$, the dominant flop count is thus $4 m n r$.

\subsection{Retractions on manifolds}

A (first-order) retraction $\mathrm{ADM}^{+} 02$ (or see [AMS08, \$4.1]) on a manifold $\mathcal{M}$ is a smooth mapping $R$ from the tangent bundle $T \mathcal{M}$ onto $\mathcal{M}$ such that

1. $R$ is defined and smooth on a neighborhood of the zero section in $T \mathcal{M}$;

2. $R(X, 0)=X$ for all $X \in \mathcal{M}$;

3. $\left.\frac{\mathrm{d}}{\mathrm{d} t} R(X, t \dot{X})\right|_{t=0}=\dot{X}$ for all $X \in \mathcal{M}$ and $\dot{X} \in \mathrm{T}_{X} \mathcal{M}$.

When $\mathcal{M}$ is an embedded submanifold of a Euclidean space $\mathcal{E}$, which is the case of the low-rank manifold $\mathcal{M}_{r}$, we say that a retraction $R$ is a second-order retraction if moreover $\left.\frac{\mathrm{d}^{2}}{\mathrm{~d} t^{2}} R(X, t \dot{X})\right|_{t=0}$ belongs to the normal space at $X$ to $\mathcal{M}$ in $\mathcal{E}$. This is one of the criteria along which the retractions described in Section 3 differ; see in particular the discussion in Section 4.2 ,

Retractions are useful in optimization algorithms for applying an update vector $\dot{X}$ to a current point $X$ [AMS08, §4] or for "lifting" an objective function to the tangent space [AMS08, §7].

In the present paper, we also consider a related concept which we call extended retraction, defined as a mapping $R$ from $\left\{\mathrm{T}_{X} \mathcal{E}: X \in \mathcal{M}\right\} \simeq\{(X, Z): X \in \mathcal{M}, Z \in \mathcal{E}\}$ onto $\mathcal{M}$ such that 
$R(X, 0)=X$ and $\left.\frac{\mathrm{d}}{\mathrm{d} t} R(X, t Z)\right|_{t=0}=\mathcal{P}_{X} Z$ for all $X \in \mathcal{M}$ and $Z \in \mathbb{R}^{m \times n}$, where $\mathcal{P}_{X}$ denotes the orthogonal projector onto the tangent space to $\mathcal{M}$ at $X$. (In the case of $\mathcal{M}_{r}, \mathcal{P}$ is given by (9).) The name "extended retraction" is justified by the fact that $R(X, Z)$ with $Z$ restricted to $\mathrm{T}_{X} \mathcal{M}$ is a retraction. This new concept will be exploited in Section 3.7 .

\section{Retractions on the low-rank manifold}

In this central section, we present and analyze several retractions on the low-rank manifold $\mathcal{M}_{r}$. At the beginning of each subsection, we mention the related literature of which we are aware. In particular, the developments in Sections 3.5 and 3.9 are new to the best of our knowledge. The Lie-Trotter retractions of Sections 3.7 and 3.8 are arguably new as (extended) retractions but they directly follow from the material in [LO13, §3.2].

\subsection{Projective retraction}

The projective retraction is perhaps the retraction that most directly comes to mind on submanifolds embedded in a Euclidean space. It consists in defining $R(X, \dot{X})$ as the projection of $X+\dot{X}$ on the manifold. The projective retraction on the low-rank manifold $\mathcal{M}_{r}$ is described and analyzed in [AM12, §3.2], and an efficient implementation is presented in Van13, §3].

The projective retraction is thus defined by

$$
R(X, \dot{X})=\underset{Y \in \mathcal{M}_{r}}{\arg \min }\|Y-(X+\dot{X})\|_{F},
$$

where $\|\cdot\|_{F}$ denotes the Frobenius norm. Let $\sigma_{1}(A), \ldots, \sigma_{\min (m, n)}(A)$ be the singular values of an $m \times n$ matrix $A$ in decreasing order. It is known [AM12, Prop. 6] that whenever $\dot{X}$ is sufficiently small for $\|\dot{X}\|<\sigma_{r}(X) / 2$ to hold, then $R(X, \dot{X})$ exists, is unique, and

$$
R(X, \dot{X})=\sum_{i=1}^{r} \sigma_{i} u_{i} v_{i}
$$

where $X+\dot{X}=\left[\begin{array}{lll}u_{1} & \ldots & u_{\min (m, n)}\end{array}\right] \operatorname{diag}\left(\sigma_{1}, \ldots, \sigma_{\min (m, n)}\right)\left[\begin{array}{lll}v_{1} & \ldots & v_{\min m, n}\end{array}\right]^{\top}$ is a singular value decomposition (SVD) with singular values in decreasing order.

If $\dot{X}$ is available in the form (12), then Van13, §3] shows that $R(X, \dot{X})$ can be computed efficiently as follows. First perform orthonormalizations $U_{p}=Q_{u} S_{u}$ and $V_{p}=Q_{v} S_{v}$. Observe that

$$
X+\dot{X}=\left[\begin{array}{ll}
U & Q_{u}
\end{array}\right]\left[\begin{array}{cc}
S+\dot{S} & S_{u} \\
S_{v}^{\top} & 0
\end{array}\right]\left[\begin{array}{c}
V^{\top} \\
Q_{v}^{\top}
\end{array}\right] .
$$

Obtain $\left(U_{s}, \Sigma_{s}, V_{s}\right)$ as an SVD (with decreasing singular values) of the small $2 r$-by- $2 r$ matrix $\left[\begin{array}{cc}S+\dot{S} & S_{u} \\ S_{v}^{\top} & 0\end{array}\right]$. Then we have

$$
R(X, \dot{X})=U_{+} S_{+} V_{+}^{\top},
$$

where $U_{+}=\left[\begin{array}{ll}U & Q_{u}\end{array}\right] U_{s}(:, 1: r), V_{+}=\left[\begin{array}{ll}V & Q_{v}\end{array}\right] V_{s}(:, 1: r)$, and $S_{+}=\Sigma_{s}(1: r, 1: r)$.

It is shown in AM12 that the projective retraction is a second-order retraction. 


\subsubsection{Matlab implementation details}

In the numerical experiments of Sections 4 and 5] this retraction is labeled proj. The orthonormalizations are obtained with the polar decomposition mentioned in Section 2.2. The SVD is computed with the svd function, which we found to be faster than getting the truncated SVD directly with svds.

\subsubsection{Flop count}

Unless otherwise stated, we assume throughout the paper that $X$ and $\dot{X}$ are given in the form (5) and (12), and we consider the dominant flop count when $r \ll m \leq n$.

The dominant flop count to compute $\left(U_{+}, S_{+}, V_{+}\right)$is then $4 m r^{2}\left[Q_{u}, S_{u}\right]+4 n r^{2}\left[Q_{v}, S_{v}\right]+$ $O\left(r^{3}\right)\left[U_{s}, \Sigma_{s}, V_{s}\right]+2 m 2 r^{2}\left[U_{+}\right]+2 n 2 r^{2}\left[V_{+}\right]$, hence $8(m+n) r^{2}$. However, one should bear in mind that the $O\left(r^{3}\right)$ flop count of the SVD concerns an operation that cannot in general be performed with finitely many elementary arithmetic operations; it thus involves an iterative process and a stopping criterion, and this may have a significant impact on the computation time when $r$ is not much smaller than $m$. This comment also applies to the matrix square root involved in the polar decomposition.

If a line search has to be performed, it is also informative to estimate the additional flop count required to compute $R(X, t \dot{X})$ for a new value of $t$. In the case of the projective retraction, this additional flop count is $O\left(r^{3}\right)\left[U_{s}, \Sigma_{s}, V_{s}\right]+2 m 2 r^{2}\left[U_{+}\right]+2 n 2 r^{2}\left[V_{+}\right]$, hence a dominant flop count of $4(m+n) r^{2}$.

\subsubsection{Inverse retraction}

Computing the inverse retraction is required in certain situations, e.g., the computation of the $R$-barycenter $X$ of a collection of points $Y_{1}, \ldots, Y_{N}$, defined by $\sum_{i=1}^{N} R_{X}^{-1} Y_{i}=0$, where $R_{X}^{-1} Y_{i}$ stands for the tangent vector $\dot{X}$ at $X$ such that $R(X, \dot{X})=Y_{i}$. Note however that, depending on the manifold and the retraction, the $R$-barycenter and the inverse retractions may not be uniquely defined.

The inverse projective retraction is given by $R_{X}^{-1} Y=\left(X+\mathrm{T}_{X} \mathcal{M}_{r}\right) \cap\left(Y+\mathrm{T}_{Y}^{\perp} \mathcal{M}_{r}\right)-X$. A rather unwieldy formula for $R_{X}^{-1} Y$ can be obtained from the expressions of the tangent space (13) and the normal space (14). In contrast, the retraction considered next has a very simple inverse, as it involves the intersection of two orthogonal affine subspaces of the embedding space $\mathbb{R}^{m \times n}$.

\subsection{Orthographic retraction}

The orthographic retraction on $\mathcal{M}_{r}$ is introduced in [AM12, §4.4], but computational aspects are not discussed therein. The concept can be found as far back as Ros61, Lue72].

The orthographic retraction $R$ on $\mathcal{M}_{r}$ is defined by setting $R(X, \dot{X})$ as the point nearest to $X+\dot{X}$ in

$$
X+\dot{X}+T_{X}^{\perp} \mathcal{M}_{r} \cap \mathcal{M}_{r} .
$$


This point is unique when $\dot{X}$ is sufficiently small. When $X$ and $\dot{X}$ are represented as in (5) and (12), $R(X, \dot{X})$ can be expressed as follows:

$$
\begin{aligned}
R(X, \dot{X}) & =\left(U(S+\dot{S})+U_{p}\right)(S+\dot{S})^{-1}\left((S+\dot{S}) V^{\top}+V_{p}^{\top}\right) \\
& =U_{+} S_{+} V_{+}^{\top},
\end{aligned}
$$

where $U(S+\dot{S})+U_{p}=: U_{+} S_{U}$ and $V\left(S^{\top}+\dot{S}^{\top}\right)+V_{p}=: V_{+} S_{V}$ are orthonormalizations and $S_{+}:=S_{U}(S+\dot{S})^{-1} S_{V}^{\top}$.

By virtue of the analysis in [AM12, this is a second-order retraction.

\subsubsection{Matlab implementation details}

This retraction is labeled orth. In our Matlab implementation, the orthonormalizations are obtained with the polar decomposition, and we use mldivide in the computation of $S_{+}$.

\subsubsection{Flop count}

Under the standing assumptions stated in Section 3.1.2, the flop count is $2 m r^{2}\left[U(S+\dot{S})+U_{p}\right]+$ $2 n r^{2}\left[V\left(S^{\top}+\dot{S}^{\top}\right)+V_{p}\right]+4 m r^{2}\left[U_{+}, S_{U}\right]+4 n r^{2}\left[V_{+}, S_{V}\right]+O\left(r^{3}\right)\left[S_{+}\right]$. The dominant cost is thus $6(m+n) r^{2}$.

The dominant additional flop count to compute $R(X, t \dot{X})$ for a new value of $t$ can be reduced to $2(m+n) r^{2}$ if adequate matrices are precomputed (namely, $U S, U \dot{S}+U_{p}, U^{\top} U_{p}, U_{p}^{\top} U_{p}, V S^{\top}$, $V \dot{S}^{\top}+V_{p}, V^{\top} V_{p}$, and $\left.V_{p}^{\top} V_{p}\right)$.

\subsubsection{Inverse retraction}

The inverse orthographic retraction is simple:

$$
R_{X}^{-1} Y=\mathcal{P}_{X}(Y-X)=Y V V^{\top}+U U^{\top} Y-U U^{\top} Y V V^{\top}-X,
$$

where $\mathcal{P}$ is the projection (9). If $Y=U_{Y} S_{Y} V_{Y}^{\top}$, we have

$$
R_{X}^{-1} Y=\left(\left(I-U U^{\top}\right) U_{Y} S_{Y} V_{Y}^{\top} V\right) V^{\top}+U\left(U^{\top} U_{Y} S_{Y} V_{Y}^{\top}\left(I-V V^{\top}\right)\right)+U\left(U^{\top} U_{Y} S_{Y} V_{Y}^{\top} V-S\right) V^{\top} .
$$

This yields the form (12) for $R_{X}^{-1} Y$.

\subsection{Quotient-based retraction: compact Stiefel approach}

A retraction that one naturally obtains by viewing $\mathcal{M}_{r}$ as the quotient (8) is the following:

$$
R(X, \dot{X})=U_{+} S_{+} V_{+}^{\top},
$$

where

$$
\begin{aligned}
U_{+} & =R_{\mathrm{St}}(U, \dot{U}) \\
S_{+} & =S+\dot{S} \\
V_{+} & =R_{\mathrm{St}}(V, \dot{V}),
\end{aligned}
$$


and $R_{\mathrm{St}}$ is a retraction on the corresponding Stiefel manifold. Since, as we have seen in (7), the decomposition (5) of $X$ is not unique, we need to ensure that the outcome $U_{+} S_{+} V_{+}^{\top}$ does not depend on the choice of the decomposition. This invariance is seen to hold if and only if the retraction on Stiefel satisfies $R_{\mathrm{St}}\left(U Q_{U}, \dot{U} Q_{U}\right)=R_{\mathrm{St}}(U, \dot{U}) Q_{U}$ for all $Q_{U} \in \mathrm{O}(r)$. One such retraction is the projective retraction on Stiefel [AM12, §3.3], advocated in [MS14, (7)], which returns the orthonormal factor of the polar decomposition of $U+\dot{U}$.

\subsubsection{Matlab implementation details}

We use the projective retraction on Stiefel for $R_{\mathrm{St}}$. The resulting retraction on $\mathcal{M}_{r}$ is thus the one of [MS14, (7)], which we label StRSt-pj.

\subsubsection{Flop count}

Assuming as usually that $\dot{X}$ is provided in the representation (12), the dominant flop count is $2 m r^{2}\left[U+U_{p} S^{-1}\right]+4 m r^{2}\left[U_{+}\right]+2 n r^{2}\left[V+V_{p} S^{-T}\right]+4 n r^{2}\left[V_{+}\right]$, that is, $6(m+n) r^{2}$.

The dominant flop count to compute $R(X, t \dot{X})$ for a new $t$ can be reduced to $2(m+n) r^{2}$ if adequate matrices are precomputed.

\subsubsection{Inverse retraction}

Assume that the projective retraction is used on Stiefel. Given $X=U S V^{\top}$ and $Y=U_{+} S_{+} V_{+}^{\top}$ in $\mathcal{M}_{r}$, we seek $\dot{X} \in \mathrm{T}_{X} \mathcal{M}_{r}$ such that $R(X, \dot{X})=Y$. Observe that $Y=\left(U_{+} Q_{U}\right)\left(Q_{U}^{\top} S_{+} Q_{V}\right)\left(V_{+} Q_{V}\right)^{\top}$ for all $Q_{U}, Q_{V}$ orthogonal. We need $U_{+} Q_{U}=(U+\dot{U}) P_{U}$ with $P_{U}$ symmetric positive definite, and this yields $U_{+}^{\top} U=: Q_{U} P_{U}$ (polar decomposition) and $\dot{U}=U_{+} Q_{U} P_{U}^{-1}-U$. Likewise, we set $V_{+}^{\top} V=: Q_{V} P_{V}$ (polar decomposition) and $\dot{V}=V_{+} Q_{V} P_{V}^{-1}-V$. Finally, $\dot{S}=Q_{U}^{\top} S_{+} Q_{V}-S$, and we have

$$
R_{U S V^{\top}}^{-1}(\dot{X})=U_{+} S_{+} V_{+}^{\top},
$$

where $\dot{X}$ is given by (11).

\subsection{Quotient-based retraction: noncompact Stiefel approach}

Yet another possibility is to define

$$
\begin{aligned}
R(X, \dot{X}) & =(U+\dot{U})(S+\dot{S})(V+\dot{V})^{\top} \\
& =\left(U S+U_{p}\right) S^{-1}(S+\dot{S}) S^{-1}\left(V S^{\top}+V_{p}\right)^{\top} \\
& =U_{+} S_{+} V_{+}^{\top},
\end{aligned}
$$

where $U S+U_{p}=U_{+} S_{U}$ and $V S^{\top}+V_{p}=V_{+} S_{V}$ are two orthonormalizations and $S_{+}=S_{U} S^{-1}(S+$ $\dot{S}) S^{-1} S_{V}^{\top}$. This is a retraction that one naturally obtains by viewing $\mathcal{M}_{r}$ as the quotient manifold $\left(\mathbb{R}_{*}^{m \times r} \times \mathrm{GL}(r) \times \mathbb{R}_{*}^{n \times r}\right) /(\mathrm{GL}(r) \times \mathrm{GL}(r))$ and favoring representations where the first and third factors are orthonormal.

\subsubsection{Matlab implementation details}

This retraction is labeled RRR. We use the polar decomposition for the orthonormalizations. 


\subsubsection{Flop count}

Assuming that the orthonormalizations are chosen as polar decompositions, the dominant flop count is $2 m r^{2}\left[U S+U_{p}\right]+4 m r^{2}\left[U_{+}, S_{U}\right]+2 n r^{2}\left[V S^{\top}+V_{p}\right]+4 n r^{2}\left[V_{+}, S_{+}\right]$, that is, $6(m+n) r^{2}$.

The dominant flop count to compute $R(X, t \dot{X})$ for a new $t$ is reduced to $4(m+n) r^{2}$ if $U S$ and $V S^{\top}$ are precomputed, and even $2(m+n) r^{2}$ if $U^{\top} \dot{U}, \dot{U}^{\top} \dot{U}, V^{\top} \dot{V}$, and $\dot{V}^{\top} \dot{V}$ are also precomputed.

\subsubsection{Inverse retraction}

We seek $\dot{X}$ in the form (11) such that $R_{U S V^{\top}}^{-1}(\dot{X})=U_{+} S_{+} V_{+}^{\top}$. Observe that $U_{+} S_{+} V_{+}^{\top}=$ $\left(U_{+} S_{U}\right)\left(S_{U}^{-1} S_{+} S_{V}^{-T}\right)\left(V_{+} S_{V}\right)^{\top}$ for all $S_{U}, S_{V}$ invertible. We thus require $U+\dot{U}=U_{+} S_{U}, S+\dot{S}=$ $S_{U}^{-1} S_{+} S_{V}^{-T}$, and $V+\dot{V}=V_{+} S_{V}$. This yields $S_{U}=\left(U^{\top} U_{+}\right)^{-1}, \dot{U}=U_{+} S_{U}-U, S_{V}=\left(V^{\top} V_{+}\right)^{-1}$, $\dot{V}=V_{+} S_{V}-V, \dot{S}=S_{U}^{-1} S_{+} S_{V}^{-T}-S$.

\subsection{Simple second-order retractions}

The retractions proposed in this section are more conveniently derived using a two-factor approach. Without loss of generality, following the notation of AAM14, we thus consider $X=M N^{\top} \in \mathcal{M}_{r}$ and $\dot{X}=M H N^{\top}+M_{\perp} K N^{\top}+M L N_{\perp}^{\top} \in \mathrm{T}_{X} \mathcal{M}_{r}$, where $M \in \mathbb{R}_{*}^{m \times r}, N \in \mathbb{R}_{*}^{n \times r}, H \in \mathbb{R}^{r \times r}$, $K \in \mathbb{R}^{(m-r) \times r}, L \in \mathbb{R}^{r \times(n-r)}$, the columns of $M_{\perp}$ form a basis of the orthogonal complement of the column space of $M$, and likewise for $N_{\perp}$ with $N$. Our goal is to derive an expression for $R(X, \dot{X})$ that makes $R$ is a second-order retraction on $\mathcal{M}_{r}$.

We seek $R$ in the form

$$
R(X, \dot{X})=\left[\begin{array}{ll}
M & M_{\perp}
\end{array}\right]\left[\begin{array}{l}
A_{0}+A_{1}+A_{2} \\
B_{0}+B_{1}+B_{2}
\end{array}\right]\left[\begin{array}{ll}
C_{0}+C_{1}+C_{2} & D_{0}+D_{1}+D_{2}
\end{array}\right]\left[\begin{array}{c}
N^{\top} \\
N_{\perp}^{\top}
\end{array}\right],
$$

where terms indexed by $j(j=0,1,2)$ are $j$ th order expressions of $\dot{X}$. The "0th order" condition on $R$ (i.e., $R(X, 0)=X$ ) yields

$$
\begin{aligned}
& A_{0} C_{0}=I \\
& B_{0} C_{0}=0 \\
& A_{0} D_{0}=0 \\
& B_{0} D_{0}=0 .
\end{aligned}
$$

The first-order condition on $R$ (i.e., $\left.\frac{\mathrm{d}}{\mathrm{d} t} R(X, t \dot{X})\right|_{t=0}=\dot{X}$ ) yields

$$
\begin{aligned}
& A_{1} C_{0}+A_{0} C_{1}=H \\
& B_{0} C_{1}+B_{1} C_{0}=K \\
& A_{0} D_{1}+A_{1} D_{0}=L \\
& B_{0} D_{1}+B_{1} D_{0}=0 .
\end{aligned}
$$


Finally, the second-order condition on $R$ (i.e., $\left.\left.\frac{\mathrm{d}^{2}}{\mathrm{~d} t^{2}} R(X, t \dot{X})\right|_{t=0} \in T_{X}^{\perp} \mathcal{M}_{r}\right)$ yields

$$
\begin{aligned}
& A_{0} C_{2}+A_{1} C_{1}+A_{2} C_{0}=0 \\
& B_{0} C_{2}+B_{1} C_{1}+B_{2} C_{0}=0 \\
& A_{0} D_{2}+A_{1} D_{1}+A_{2} D_{0}=0 \\
& B_{0} D_{2}+B_{1} D_{1}+B_{2} D_{0} \text { arbitrary. }
\end{aligned}
$$

The above system of matrix equations is underdetermined. A simple solution is readily found to be

$$
\begin{gathered}
A_{0}=C_{0}=I, B_{0}=D_{0}=0, \\
A_{1}=H, C_{1}=0, B_{1}=K, D_{1}=L, \\
A_{2}=0, C_{2}=0, B_{2}=0, D_{2}=-H L .
\end{gathered}
$$

The resulting retraction is thus given by

$$
R(X, \dot{X})=\left[\begin{array}{ll}
M & M_{p}
\end{array}\right]\left[\begin{array}{c}
I+H \\
I
\end{array}\right]\left[\begin{array}{ll}
I & I-H
\end{array}\right]\left[\begin{array}{l}
N^{\top} \\
N_{p}^{\top}
\end{array}\right],
$$

where we set $M_{p}=M_{\perp} K$ and $N_{p}=N_{\perp} L^{\top}$.

In the three-factor representation (5) and (12), this yields

$$
R(X, \dot{X})=U_{+} S_{+} V_{+}^{\top}
$$

where $U_{+} S_{U}:=U(S+\dot{S})+U_{p}$ and $V_{+} S_{V}:=V+V_{p} S^{-T}\left(I-\dot{S}^{\top} S^{-T}\right)$ are orthonormalizations and $S_{+}:=S_{U} S_{V}^{\top}$. To see this, link the 2-factor and 3-factor representations by taking $M=U$ and $N=V S^{\top}$; the relation between the tangent vector representations is then seen to be given by $\dot{S}=H S, U_{p}=M_{\perp} K S, V_{p}=N_{\perp} L^{\top}$, and the retraction then writes

$$
R(X, \dot{X})=\left[\begin{array}{ll}
U & U_{p} S^{-1}
\end{array}\right]\left[\begin{array}{c}
I+\dot{S} S^{-1} \\
I
\end{array}\right]\left[\begin{array}{ll}
S & I-\dot{S} S^{-1}
\end{array}\right]\left[\begin{array}{c}
V^{\top} \\
V_{p}^{\top}
\end{array}\right]
$$

\subsubsection{Matlab implementation details}

This retraction is labeled ez-2nd. We use the polar decomposition for the orthonormalizations. We explicitly compute $S^{-1}$ using inv as it appears twice.

\subsubsection{Flop count}

Assuming as usually that $X$ as in (5) and $\dot{X}$ as in (12) are provided, the dominant flop count is $2 m r^{2}\left[U(S+\dot{S})+U_{p}\right]+2 n r^{2}\left[V+V_{p} S^{-T}\left(I-\dot{S}^{\top} S^{-T}\right)\right]+4 m r^{2}\left[U_{+}\right]+4 n r^{2}\left[V_{+}\right]$, hence $6(m+n) r^{2}$.

The dominant flop count to compute $R(X, t \dot{X})$ for a new $t$ can be reduced to $2(m+n) r^{2}$ with adequate precomputed matrices. 


\subsection{Simple second-order balanced retraction}

In the underdetermined system of equations obtained in Section 3.5, if we moreover impose a better balancing between the left and right factors by further requiring that $A_{1}=C_{1}$, then we are led to the retraction proposed in [SWC13, Lemma 4]. In the representation (5) and (12), it writes as follows:

$$
R(X, \dot{X})=U_{+} S_{+} V_{+}^{\top}
$$

where $U_{+} S_{U}:=U\left(S+\frac{1}{2} \dot{S}-\frac{1}{8} \dot{S} S^{-1} \dot{S}\right)+U_{p}\left(I-\frac{1}{2} S^{-1} \dot{S}\right)$ and $V_{+} S_{V}:=V\left(S^{\top}+\frac{1}{2} \dot{S}^{\top}-\frac{1}{8} \dot{S}^{\top} S^{-T} \dot{S}^{\top}\right)+$ $V_{p}\left(I-\frac{1}{2} S^{-T} \dot{S}^{\top}\right)$ are orthonormalizations and $S_{+}:=S_{U} S^{-1} S_{V}^{\top}$.

\subsubsection{Matlab implementation details}

This retraction is labeled Shalit. The same comments as those of Section 3.5 apply.

\subsubsection{Flop count}

The dominant flop count is the same as in Section 3.5

\subsection{Lie-Trotter extended retraction}

Observe that the three terms in $\mathcal{P}_{X} Z$ (9) belong to $\mathrm{T}_{X} \mathcal{M}_{R}$. Following [013, §3.2], let us define the KSL Lie-Trotter extended retraction $R$ on $\mathcal{M}_{r}$ by $\operatorname{setting} R(X, Z)$ as follows for all $Z \in \mathrm{T}_{X} \mathbb{R}^{m \times n} \simeq \mathbb{R}^{m \times n}$, thus in particular for all $Z \in \mathrm{T}_{X} \mathcal{M}_{r}$ :

1. Obtain $U_{1}$ and $\hat{S}_{1}$ from

$$
U_{1} \hat{S}_{1} V^{\top}=U S V^{\top}+Z V V^{\top}
$$

One gets $U_{1}$ and $\hat{S}_{1}$ by an orthonormalization $U_{1} \hat{S}_{1}=U S+Z V$ with $U_{1}$ orthonormal.

2. Obtain $\tilde{S}_{0}$ from

$$
U_{1} \tilde{S}_{0} V^{\top}=U_{1} \hat{S}_{1} V^{\top}-U_{1} U_{1}^{\top} Z V V^{\top}
$$

One gets $\tilde{S}_{0}$ by $\tilde{S}_{0}=\hat{S}_{1}-U_{1}^{\top} Z V$.

3. Obtain $V_{1}$ and $S_{1}$ from

$$
U_{1} S_{1} V_{1}^{\top}=U_{1} \tilde{S}_{0} V^{\top}+U_{1} U_{1}^{\top} Z
$$

One gets $V_{1}$ and $S_{1}$ by an orthonormalization $V_{1} S_{1}^{\top}=V \tilde{S}_{0}^{\top}+Z^{\top} U_{1}$.

Finally, set

$$
R(X, Z)=U_{1} S_{1} V_{1}^{\top}
$$

\subsubsection{Analysis}

We now need to show that the above procedure indeed defines an extended retraction on $\mathcal{M}_{r}$.

First, it can be shown that $R(X, Z)$ is well defined, i.e., the outcome (25) does not depend on the choice of the representation (5) of $X$ nor on the orthonormalizations that yield $U_{1}$ and $V_{1}$. To see this, consider the above procedure carried out with two representations $X=U S V^{\top}=\underline{U S V}{ }^{\top}$, 
and use the underline notation to denote the results obtained with the second representation. One has, for some $Q_{U}, Q_{V} \in \mathrm{O}(r), \underline{U}=U Q_{U}, \underline{V}=V Q_{V}$, and $\underline{S}=Q_{U}^{\top} S Q_{V}$. We then find that, for some $Q_{U_{1}}, Q_{V_{1}} \in \mathrm{O}(r)$, we have $\underline{U}_{1}=U Q_{U_{1}}, \underline{\hat{S}}_{1}=U_{U_{1}}^{\top} \hat{S}_{1} Q_{V}, \underline{\tilde{S}}_{0}=Q_{U_{1}}^{\top} \tilde{S}_{0} Q_{V}, \underline{V}_{1}=V_{1} Q_{V_{1}}$, and $\underline{\tilde{S}}_{1}=Q_{U_{1}}^{\top} S_{1} Q_{V_{1}}$, which yields that $U_{1} S_{1} V_{1}^{\top}=\underline{U}_{1} \underline{S}_{1} \underline{V}_{1}^{\top}$. For example, the first step yields $U_{1} \hat{S}_{1}=$ $U S+Z V$ and $\underline{U}_{1} \underline{\hat{S}}_{1}=\underline{U S}+Z \underline{V}$, which yields the above relations $\underline{U}_{1}=U Q_{U_{1}}, \underline{\hat{S}}_{1}=U_{U_{1}}^{\top} \hat{S}_{1} Q_{V}$.

Second, the mapping $R$ is smooth. This is readily seen by choosing the polar decomposition for the orthonormalizations and noting that the polar factors are smooth functions of their product.

Third, it is readily checked that the zeroth-order property holds: $R(X, 0)=X$ for all $X \in \mathcal{M}_{r}$.

The forth and final point is to show the first order property: $\left.\frac{\mathrm{d}}{\mathrm{d} t} R(X, t Z)\right|_{t=0}=\mathcal{P}_{X} Z$ for all $X \in \mathcal{M}$ and $Z \in \mathbb{R}^{m \times n}$. Since $R(X, t Z)$ amounts to the KSL integration of the low-rank ordinary differential equation $\dot{Y}(t)=\mathcal{P}_{Y(t)} Z$ with initial condition $Y(0)=X$, the first order property follows directly from the fact that KSL is a consistent integrator. Alternatively, the property can be proved from basic principles as follows. First observe that if $U(t) S(t)=A(t)$ is a time-varying polar decomposition, then $U^{\prime}=\left(I-U U^{\top}\right) A^{\prime} S^{-1}+U$ skew $\left(U^{\top} A^{\prime}\right) S^{-1}$ and $S^{\prime}=\operatorname{sym}\left(U^{\top} A^{\prime}\right)$, where $\operatorname{skew}(A)=\frac{1}{2}\left(A-A^{\top}\right)$ and $\operatorname{sym}(A)=\frac{1}{2}\left(A+A^{\top}\right)$ denote the skew-symmetric and symmetric components of $A$. This can be deduced using the product rule and the expression $\mathrm{T}_{U} \operatorname{St}(m, r)=$ $\left\{U \Omega+U_{\perp} K: \Omega=-\Omega^{\top} \in \mathbb{R}^{r \times r}, K \in \mathbb{R}^{(m-r) \times r}\right\}$ where $U_{\perp}$ is such that $\left[\begin{array}{ll}U & U_{\perp}\end{array}\right] \in \mathrm{O}(m)$. We then obtain $\left.\frac{\mathrm{d}}{\mathrm{d} t} U_{1}\right|_{t=0}=\left(I-U U^{\top}\right) Z V S^{-1}+U \operatorname{skew}\left(U^{\top} Z V\right) S^{-1},\left.\frac{\mathrm{d}}{\mathrm{d} t} \hat{S}_{1}\right|_{t=0}=\operatorname{sym}\left(U^{\top} Z V\right)$, $\left.\frac{\mathrm{d}}{\mathrm{d} t} \tilde{S}_{0}\right|_{t=0}=\operatorname{sym}\left(U^{\top} Z V\right)-U^{\top} Z V=-\operatorname{skew}\left(U^{\top} Z V\right),\left.\frac{\mathrm{d}}{\mathrm{d} t} V_{1}\right|_{t=0}=\left(I-V V^{\top}\right)\left(V \operatorname{skew}\left(U^{\top} Z V\right)+\right.$ $\left.Z^{\top} U\right) S^{-T}+V \operatorname{skew}\left(V^{\top}\left(V \operatorname{skew}\left(U^{\top} Z V\right)+Z^{\top} U\right)\right) S^{-T}=\left(I-V V^{\top}\right) Z^{\top} U S^{-T}$, and $\left.\frac{\mathrm{d}}{\mathrm{d} t} S_{1}^{\top}\right|_{t=0}=$ $\operatorname{sym}\left(V^{\top}\left(V \operatorname{skew}\left(U^{\top} Z V\right)+Z^{\top} U\right)\right)=\operatorname{sym}\left(V^{\top} S U\right)$. This yields $\left.\frac{\mathrm{d}}{\mathrm{d} t} U_{1} S_{1} V_{1}^{\top}\right|_{t=0}=\left.\frac{\mathrm{d}}{\mathrm{d} t} U_{1}\right|_{t=0} S V^{\top}+$ $\left.U \frac{\mathrm{d}}{\mathrm{d} t} S_{1}\right|_{t=0} V^{\top}+\left.U S \frac{\mathrm{d}}{\mathrm{d} t} V_{1}^{\top}\right|_{t=0}=\mathcal{P}_{X} Z$.

We have thus shown the following.

Proposition 3.1 (Lie-Trotter extended retraction). The $R$ mapping defined by (25) is a welldefined extended retraction on $\mathcal{M}_{r}$.

Note that, since $U_{1}$ appears instead of $U$ in (23) and (24), the component of $Z$ in the normal space $T_{X}^{\perp} \mathcal{M}_{r}$ has an impact on the outcome (25); that is, $R(X, Z) \neq R\left(X, \mathcal{P}_{X} Z\right)$ in general.

We now proceed to show that the Lie-Trotter extended retraction is a second-order retraction. The proof will make use of the following remarkable property of the Lie-Trotter extended retraction, which can be deduced directly from [LO13, Theorem 4.1].

Proposition 3.2 (exactness property). Let $R$ be the Lie-Trotter extended retraction defined in (25). For all $X, Y \in \mathcal{M}_{r}$, it holds that $R(X, Y-X)=Y$.

Note that the expression $R(X, Y-X)$ only makes sense for extended retractions, as $Y-X$ does in general not belong to $\mathrm{T}_{X} \mathcal{M}_{r}$.

Theorem 3.3 (second-order property). Let $R$ be the Lie-Trotter extended retraction defined in (25). For all $X \in \mathrm{T}_{X} \mathcal{M}_{r}$ and all $\dot{X} \in \mathrm{T}_{X} \mathcal{M}_{r}$, it holds that $R(X, t \dot{X})=R_{\text {ortho }}(X, t \dot{X})+O\left(t^{3}\right)$. Since $R_{\text {ortho }}$ is a second-order retraction, it follows that $R$ is a second-order retraction.

Proof. We freely drop the foot $X$ in the notation. Observe that $R_{\text {ortho }}(t \dot{X})=X+t \dot{X}+O_{\mathrm{N}}\left(t^{2}\right)$ 
where $O_{\mathrm{N}}\left(t^{2}\right) \in T_{X}^{\perp} \mathcal{M}_{r}$ with $\left\|O_{\mathrm{N}}\left(t^{2}\right)\right\| \leq c t^{2}$ for all $t$ sufficiently small. This yields

$$
\begin{aligned}
R(t \dot{X}) & =R\left(R_{\text {ortho }}(t \dot{X})-X+O_{\mathrm{N}}\left(t^{2}\right)\right) \\
& =R\left(R_{\text {ortho }}(t \dot{X})-X\right)+\mathrm{D} R\left(R_{\text {ortho }}(t \dot{X})-X\right)\left[O_{\mathrm{N}}\left(t^{2}\right)\right]+O\left(t^{4}\right) \\
& =R\left(R_{\text {ortho }}(t \dot{X})-X\right)+(\mathrm{D} R(0)+O(t))\left[O_{\mathrm{N}}\left(t^{2}\right)\right]+O\left(t^{4}\right) \\
& =R\left(R_{\text {ortho }}(t \dot{X})-X\right)+O\left(t^{3}\right)
\end{aligned}
$$

since $\mathrm{D} R(0)$ is the projection onto the tangent space,

$$
=R_{\text {ortho }}(t \dot{X})+O\left(t^{3}\right)
$$

by the exactness property.

\subsubsection{Flop count}

Assuming that $Z$ is a full $m \times n$ matrix and $X$ is available in the factorized form (5), the dominant cost is $2 m r^{2}+2 m n r[U S+Z V]+4 m r^{2}\left[U_{1}, \hat{S}_{1}\right]+2 m r^{2}\left[U_{1}^{\top} Z V\right]+r^{2}\left[\hat{S}_{1}-U_{1}^{\top} Z V\right]+2 n r^{2}+2 m n r\left[V \tilde{S}_{0}^{\top}+\right.$ $\left.Z^{\top} U_{1}\right]+4 n r^{2}\left[V_{1}, S_{1}^{\top}\right]$, hence $4 m n r+8 m r^{2}+6 n r^{2}$.

Note however that this extended retraction does not play in the same league as the other retractions mentioned above, as its input is a full $m \times n$ matrix $Z$ instead of an $\dot{X}$ represented as in (12). Recall from Section 2.2 that computing $\dot{X}$ as $\mathcal{P}_{X} Z$ already requires $4 m n r+4 m r^{2}+2 n r^{2}$ flops.

The dominant additional flop count to compute $R(X, t Z)$ for a new value of $t$ is $4 m r^{2}\left[U_{1}, \hat{S}_{1}\right]+$ $2 m r^{2}\left[t U_{1}^{\top} Z V\right]+r^{2}\left[\hat{S}_{1}-t U_{1}^{\top} Z V\right]+2 m n r\left[V \tilde{S}_{0}^{\top}+t Z^{\top} U_{1}\right]+4 n r^{2}\left[V_{1}, S_{1}^{\top}\right]$. In comparison, for each retraction $\tilde{R}$ defined above, computing $\tilde{R}\left(X, t \mathcal{P}_{X} Z\right)$ for a new $t$ has a cost of $O\left((m+n) r^{2}\right)$ only, since $\mathcal{P}_{X} Z$ can be precomputed. The Lie-Trotter extended retraction is thus not competitive in the "new $t$ " scenario.

\subsection{Lie-Trotter retraction}

The (KSL) Lie-Trotter retraction $R$ is simply defined as the Lie-Trotter extended retraction (25) where $Z$ is restricted to belong to $\mathrm{T}_{X} \mathcal{M}_{r}$. The sole purpose of this section is to present a computationally efficient way of computing $R(X, \dot{X})$ when $\dot{X}$ is available in the $\left(U_{p}, S, V_{p}\right)$ form (12).

1. Get $U_{1}$ and $\hat{S}_{1}$ by an orthonormalization $U_{1} \hat{S}_{1}=U(S+\dot{S})+U_{p}$ with $U_{1}$ orthonormal.

2. Get $\tilde{S}_{0}$ by $\tilde{S}_{0}=\hat{S}_{1}-\left(U_{1}^{\top} U_{p}+\left(U_{1}^{\top} U\right) \dot{S}\right)$.

3. Get $V_{1}$ and $S_{1}$ by an orthonormalization $V_{1} S_{1}^{\top}=V \tilde{S}_{0}^{\top}+Z^{\top} U_{1}$, with $Z$ as in (12).

Finally, set

$$
R(X, \dot{X})=U_{1} S_{1} V_{1}^{\top} .
$$

This is a second-order retraction in view of the analysis in Section 3.7 


\subsubsection{Matlab implementation details}

This retraction is labeled KSL. We use the polar decomposition for the orthonormalizations. From the first step, we have $U^{\top} U_{1}=(S+\dot{S}) \hat{S}_{1}^{-1}$. We compute $V \tilde{S}_{0}^{\top}+Z^{\top} U_{1}$ as $V\left(\tilde{S}_{0}^{\top}+U_{p}^{\top} U_{1}+\right.$ $\left.\dot{S}^{\top}\left(U^{\top} U_{1}\right)\right)+V_{p}\left(U^{\top} U_{1}\right)$.

\subsubsection{Flop count}

The dominant flop count is $2 m r^{2}\left[U(S+\dot{S})+U_{p}\right]+4 m r^{2}\left[U_{1}, \hat{S}_{1}\right]+O\left(r^{3}\right)\left[U^{\top} U_{1}\right]+2 m r^{2}\left[U_{1}^{\top} U_{p}\right]+$ $4 n r^{2}\left[V \tilde{S}_{0}^{\top}+Z^{\top} U_{1}\right]+4 n r^{2}\left[V_{1}, S_{1}^{\top}\right]$, that is, $8(m+n) r^{2}$.

The dominant flop count to compute $R(X, t \dot{X})$ for a new $t$ can be reduced to $4 m r^{2}+6 n r^{2}$.

\subsection{Modified Lie-Trotter retraction}

Observe that the above procedure adds $\dot{S}$ twice and subtracts it once. Instead, one could modify the procedure as follows to add it only once:

1. Get $U_{1}$ and $\hat{S}_{1}$ by a decomposition $U_{1} \hat{S}_{1}=U S+U_{p}$ with $U_{1}$ orthonormal.

2. Get $\tilde{S}_{0}$ by $\tilde{S}_{0}=\hat{S}_{1}+U_{1}^{\top} U \dot{S}$.

3. Get $V_{1}$ and $S_{1}$ by a decomposition $V_{1} S_{1}^{\top}=V \tilde{S}_{0}^{\top}+V_{p} U^{\top} U_{1}$.

Finally, set

$$
R(X, \dot{X})=U_{1} S_{1} V_{1}^{\top} .
$$

This can be shown to be a retraction using techniques similar to those employed in Section 3.7 However, numerical experiments indicate that this is not a second-order retraction; see Section 4.2 .

\subsubsection{Matlab implementation details}

This retraction is labeled KSL+. In view of the first step, the product $U^{\top} U_{1}$ appearing in steps 2 and 3 can be computed as $S \hat{S}_{1}^{-1}$.

\subsubsection{Flop count}

The dominant flop count is $2 m r^{2}\left[U S+U_{p}\right]+4 m r^{2}\left[U_{1}, \hat{S}_{1}\right]+4 n r^{2}\left[V \tilde{S}_{0}^{\top}+V_{p} U^{\top} U_{1}\right]+4 n r^{2}\left[V_{1}, S_{1}^{\top}\right]$, thus $6 m r^{2}+8 n r^{2}$. When $m<n$, the "LSK" way (computing $V_{1}$ first) is preferable in terms of flops; this amounts to the (different) retraction that maps $(X, \dot{X})$ to $R\left(X^{\top}, \dot{X}^{\top}\right)^{\top}$, with dominant flop count of $8 m r^{2}+6 n r^{2}$. We use the LSK way in our experiments when $m<n$.

The dominant flop count to compute $R(X, t \dot{X})$ for a new $t$ can be reduced to $2 m r^{2}+6 n r^{2}$ if adequate matrix products are precomputed.

\subsection{Exponential retraction}

The exponential retraction is defined by $R(X, \dot{X})=\gamma(1)$, where $\gamma$ is the geodesic on $\mathcal{M}_{r}$ (viewed as a Riemannian submanifold of $\mathbb{R}^{m \times n}$ ) with initial conditions $\gamma(0)=X$ and $\gamma^{\prime}(0)=\dot{X}$. The exponential is arguably the "theoretically ideal" retraction, but it was realized early on that 
trying to move along geodesics is usually computationally expensive Lue72. Formalizing the idea of resorting instead to first-order approximations of geodesics was a motivation behind the concept of retraction Shu86, $\mathrm{ADM}^{+} 02$. And indeed, for our manifold $\mathcal{M}_{r}$, we are not aware of a closed-form expression for the geodesics. (Note however that there is a Riemannian metric on $\mathcal{M}_{r}$, different from the embedded metric, for which geodesics admit a closed-form expression; see [AAM14, §6.11].)

Nevertheless, it is worthwhile investigating how the retractions proposed above compare with the exponential. To this end, we have implemented the following basic numerical scheme for solving the geodesic equation $\mathcal{P}_{X(t)} X^{\prime \prime}(t)=0$, where $\mathcal{P}$ is the tangent projection (9): $X(t+\delta)=$ $R_{\text {ortho }}(X(t), \delta \dot{X}(t)), \dot{X}(t+\delta)=\mathcal{P}_{X(t+\delta)} \dot{X}(t)$. As usually in numerical integration schemes, the choice of $\delta$ is guided by the conflicting goals of achieving low truncation errors, rounding errors, and computation times. In our numerical experiments, we found that $\delta=10^{-3}$ was an acceptable compromise, and we did not attempt to choose $\delta$ adaptively.

This retraction is labeled geod.

\section{Numerical tests}

We now compare numerically the various retractions described above. The Matlab code that generated the tables is available from http://sites.uclouvain.be/absil/2013.04.

\subsection{Pairwise distances}

In a first set of experiments reported in Table $\square$ we compute the pairwise distances $\| R_{i}(X, t \dot{X})-$ $R_{j}(X, t \dot{X}) \|_{F}$, where $R_{i}$ stands for the $i$ th retraction in our list. Matrix $X$ is represented as in (5) , where $U$ and $V$ are generated by orthonormalizing matrices drawn from the standard normal distribution and $S$ is drawn from the standard normal distribution. The tangent vector $\dot{X}=\mathcal{P}_{X} Z$ is generated by drawing an $m \times n$ matrix $Z$ from the standard normal distribution. For this small value of $t$, one observes that the second-order retractions $R$ (i.e., all the retractions but StRSt-pj, $\mathrm{RRR}$, and $\mathrm{KSL}+)$ achieve the smaller distance between $R(X, t \dot{X})$ and $R_{\text {geod }}(X, t \dot{X})$.

Table 1: Pairwise distances with $m=1.0 \mathrm{e}+03, n=1.0 \mathrm{e}+03, r=1.0 \mathrm{e}+01, t=1.0 \mathrm{e}-04$.

\begin{tabular}{|c|c|c|c|c|c|c|c|c|c|}
\hline & proj & ortho & StRSt_pj & RRR & $e z-2 n d$ & Shalit & KSL & $\mathrm{KSL}+$ & geod \\
\hline proj & $0.0 e+00$ & $1.3 e-05$ & $1.8 e-04$ & $1.9 e-05$ & $1.3 e-05$ & $1.3 e-05$ & $9.2 e-06$ & $1.6 e-05$ & $8.8 e-06$ \\
\hline ortho & $1.3 e-05$ & $0.0 e+00$ & $1.8 e-04$ & $2.1 e-05$ & $3.4 \mathrm{e}-08$ & $1.6 e-08$ & $9.5 e-06$ & $1.9 e-05$ & $4.4 e-06$ \\
\hline StRSt_pj & $1.8 e-04$ & $1.8 e-04$ & $0.0 e+00$ & $1.7 e-04$ & $1.8 e-04$ & $1.8 e-04$ & $1.8 e-04$ & $1.7 e-04$ & $1.8 e-04$ \\
\hline RRR & $1.9 e-05$ & $2.1 e-05$ & $1.7 e-04$ & $0.0 e+00$ & $2.1 e-05$ & $2.1 e-05$ & $2.0 e-05$ & $1.1 e-05$ & $2.0 e-05$ \\
\hline$e z-2 n d$ & $1.3 e-05$ & $3.4 e-08$ & $1.8 e-04$ & $2.1 e-05$ & $0.0 e+00$ & $2.3 e-08$ & $9.5 e-06$ & $1.9 e-05$ & $4.4 e-06$ \\
\hline Shalit & $1.3 e-05$ & $1.6 e-08$ & $1.8 e-04$ & $2.1 e-05$ & $2.3 e-08$ & $0.0 e+00$ & $9.5 e-06$ & $1.9 e-05$ & $4.4 e-06$ \\
\hline KSL & $9.2 e-06$ & $9.5 e-06$ & $1.8 e-04$ & $2.0 e-05$ & $9.5 e-06$ & $9.5 e-06$ & $0.0 e+00$ & $1.8 e-05$ & $7.0 e-06$ \\
\hline KSL+ & $1.6 e-05$ & $1.9 e-05$ & $1.7 e-04$ & $1.1 e-05$ & $1.9 e-05$ & $1.9 e-05$ & $1.8 e-05$ & $0.0 e+00$ & $1.7 e-05$ \\
\hline geod & $8.8 e-06$ & $4.4 e-06$ & $1.8 e-04$ & $2.0 e-05$ & $4.4 e-06$ & $4.4 e-06$ & $7.0 e-06$ & $1.7 e-05$ & $0.0 e+00$ \\
\hline
\end{tabular}

Table 2 shows the results obtained for the same experiment but with $S$ now chosen to have a large condition number. Specifically, we choose $S$ with singular values equal to 1 ( $r-1$ times) and 
$10^{-6}$ (one time). The various retractions are seen to behave very differently in this ill-conditioned setting. In particular, a large discrepancy is observed between RRR, ez-2nd, Shalit, and the other retractions. These retractions are readily seen to be unbounded: bounded inputs do not yield bounded outputs. The orth retraction is also unbounded, but the unboundedness becomes apparent for inputs such that $S+\dot{S}$ is ill-conditioned. All the other retractions considered above are bounded, namely proj, StRSt-pj, KSL, KSL+, and geod.

Table 2: Pairwise distances with $m=1.0 \mathrm{e}+03, n=1.0 \mathrm{e}+03, r=1.0 \mathrm{e}+01, t=1.0 \mathrm{e}-04$.

\begin{tabular}{|c|c|c|c|c|c|c|c|c|c|}
\hline & proj & ortho & StRSt_pj & RRR & ez-2nd & Shalit & KSL & KSL+ & geod \\
\hline proj & $0.0 \mathrm{e}+00$ & $9.4 \mathrm{e}-02$ & $3.2 \mathrm{e}-03$ & $1.0 \mathrm{e}+03$ & $1.0 \mathrm{e}+03$ & $2.5 \mathrm{e}+04$ & $2.2 \mathrm{e}-03$ & $3.8 \mathrm{e}-03$ & $1.9 \mathrm{e}-03$ \\
ortho & $9.4 \mathrm{e}-02$ & $0.0 \mathrm{e}+00$ & $9.6 \mathrm{e}-02$ & $1.0 \mathrm{e}+03$ & $1.0 \mathrm{e}+03$ & $2.5 \mathrm{e}+04$ & $9.6 \mathrm{e}-02$ & $9.6 \mathrm{e}-02$ & $9.5 \mathrm{e}-02$ \\
StRSt_pj & $3.2 \mathrm{e}-03$ & $9.6 \mathrm{e}-02$ & $0.0 \mathrm{e}+00$ & $1.0 \mathrm{e}+03$ & $1.0 \mathrm{e}+03$ & $2.5 \mathrm{e}+04$ & $3.3 \mathrm{e}-03$ & $3.2 \mathrm{e}-03$ & $4.0 \mathrm{e}-03$ \\
RRR & $1.0 \mathrm{e}+03$ & $1.0 \mathrm{e}+03$ & $1.0 \mathrm{e}+03$ & $0.0 \mathrm{e}+00$ & $2.0 \mathrm{e}+03$ & $2.4 \mathrm{e}+04$ & $1.0 \mathrm{e}+03$ & $1.0 \mathrm{e}+03$ & $1.0 \mathrm{e}+03$ \\
ez-2nd & $1.0 \mathrm{e}+03$ & $1.0 \mathrm{e}+03$ & $1.0 \mathrm{e}+03$ & $2.0 \mathrm{e}+03$ & $0.0 \mathrm{e}+00$ & $2.6 \mathrm{e}+04$ & $1.0 \mathrm{e}+03$ & $1.0 \mathrm{e}+03$ & $1.0 \mathrm{e}+03$ \\
Shalit & $2.5 \mathrm{e}+04$ & $2.5 \mathrm{e}+04$ & $2.5 \mathrm{e}+04$ & $2.4 \mathrm{e}+04$ & $2.6 \mathrm{e}+04$ & $0.0 \mathrm{e}+00$ & $2.5 \mathrm{e}+04$ & $2.5 \mathrm{e}+04$ & $2.5 \mathrm{e}+04$ \\
KSL & $2.2 \mathrm{e}-03$ & $9.6 \mathrm{e}-02$ & $3.3 \mathrm{e}-03$ & $1.0 \mathrm{e}+03$ & $1.0 \mathrm{e}+03$ & $2.5 \mathrm{e}+04$ & $0.0 \mathrm{e}+00$ & $4.5 \mathrm{e}-03$ & $3.7 \mathrm{e}-03$ \\
KSL+ & $3.8 \mathrm{e}-03$ & $9.6 \mathrm{e}-02$ & $3.2 \mathrm{e}-03$ & $1.0 \mathrm{e}+03$ & $1.0 \mathrm{e}+03$ & $2.5 \mathrm{e}+04$ & $4.5 \mathrm{e}-03$ & $0.0 \mathrm{e}+00$ & $3.9 \mathrm{e}-03$ \\
geod & $1.9 \mathrm{e}-03$ & $9.5 \mathrm{e}-02$ & $4.0 \mathrm{e}-03$ & $1.0 \mathrm{e}+03$ & $1.0 \mathrm{e}+03$ & $2.5 \mathrm{e}+04$ & $3.7 \mathrm{e}-03$ & $3.9 \mathrm{e}-03$ & $0.0 \mathrm{e}+00$ \\
\hline
\end{tabular}

Several other experiments on pairwise distances could be conducted to get a more detailed understanding of the differences between the various retractions. In particular, since the retractions do not have the same domain of definition, pushing them to the limit of their domain of definition can reveal marked differences.

\subsection{Second-order property}

As we have seen, retractions proj, ortho, ez-2nd, Shalit, KSL, and geod are second-order retractions. In Table 3, we report an experiment that corroborates this finding and indicates that the other retractions considered above are not second-order retractions. The table provides the values of the Frobenius norm of the tangent projection of the second-order finite difference, i.e., $\delta_{i}(t):=\left\|\mathcal{P}_{X}\left(R_{i}(X, t \dot{X})-2 X+R_{i}(X,-t \dot{X})\right)\right\|_{F} / t^{2}$ where $R_{i}$ stands for the $i$ th retraction in the list. If $\delta_{i}(t)$ is $O(t)$ (and thus $O\left(t^{2}\right)$ since it is an even function), it indicates that $R_{i}$ is a secondorder retraction. If it behaves like $O(1)$, it indicates that $R_{i}$ is not a second-order retraction. It appears from Table 3 that StRSt-pj, RRR, and KSL+ are not second-order retractions, while all the other retractions are second-order retractions.

Note that $\delta(t)$ is in fact identically zero in exact arithmetic for ortho and ez-2nd. This explains why the $O(t)$ behavior is not visible in Table 3 for these two retractions. The property that $\delta(t) \equiv 0$ is obvious for ortho: in view of (15), we have that $\mathcal{P}_{X}\left(R_{\text {ortho }}(X, \dot{X})-X\right)=\dot{X}$, hence $\mathcal{P}_{X}\left(R_{\text {ortho }}(X, t \dot{X})-2 X+R_{\text {ortho }}(X,-t \dot{X})\right)=\mathcal{P}_{X}\left(R_{\text {ortho }}(X, t \dot{X})-X\right)+\mathcal{P}_{X}\left(R_{\text {ortho }}(X,-t \dot{X})-X\right)=$ $t \dot{X}-t \dot{X}=0$. For ez-2nd, one can show that $\mathcal{P}_{X}\left(R_{\mathrm{ez}-2 \mathrm{nd}}(X, t \dot{X})-X\right)$ is an odd function of $t$, and the property follows. 
Table 3: Norm of the tangent projection of the second-order finite difference.

\begin{tabular}{|c|c|c|c|c|c|c|c|c|c|}
\hline$t$ & proj & ortho & StRSt_pj & RRR & ez-2nd & Shalit & KSL & KSL+ & geod \\
\hline $1.0 \mathrm{e}-03$ & $1.2 \mathrm{e}+01$ & $2.5 \mathrm{e}-09$ & $1.8 \mathrm{e}+03$ & $5.2 \mathrm{e}+02$ & $2.1 \mathrm{e}-09$ & $1.6 \mathrm{e}-03$ & $1.9 \mathrm{e}+00$ & $1.8 \mathrm{e}+02$ & $2.1 \mathrm{e}+00$ \\
$1.0 \mathrm{e}-04$ & $1.2 \mathrm{e}-01$ & $2.6 \mathrm{e}-07$ & $1.8 \mathrm{e}+03$ & $5.2 \mathrm{e}+02$ & $2.1 \mathrm{e}-07$ & $1.6 \mathrm{e}-05$ & $1.9 \mathrm{e}-02$ & $1.8 \mathrm{e}+02$ & $2.2 \mathrm{e}-02$ \\
\hline
\end{tabular}

\subsection{Symmetry}

Most of the retractions presented above are readily seen to preserve symmetry, i.e., $R(X, \dot{X})$ is a symmetric matrix if $X$ and $\dot{X}$ are symmetric matrices. The exceptions are ez-2nd, KSL, and KSL+. The experiments reported in Table 4 confirm that these three retractions do not preserve symmetry. In these experiments, $X$ and $\dot{X}$ are chosen symmetric and the Frobenius norm of $R(X, \dot{X})-R(X, \dot{X})^{\top}$ is computed.

Table 4: Symmetry test.

\begin{tabular}{|c|c|c|c|c|c|c|c|c|}
\hline proj & ortho & StRSt_pj & RRR & ez-2nd & Shalit & KSL & KSL+ & geod \\
\hline $6.9 e-14$ & $1.0 e-13$ & $4.9 e-14$ & $8.3 e-14$ & $2.1 e+00$ & $9.9 e-14$ & $1.1 e+01$ & $1.1 e+01$ & $1.3 e-12$ \\
\hline
\end{tabular}

\subsection{Run times}

For information, we report wall-clock computation times for $X$ of square shape (Table 5) and for $X$ of horizontal shape (Table 6). The tests were run with MATLAB Version 7.13.0.564 (R2011b) on a PC with two Intel(R) Pentium(R) D CPU 3.00GHz, $2048 \mathrm{~KB}$ cache each, running Linux kernel 3.2.0. The timings observed are compatible with the flop counts mentioned for the various retractions, and geod, as expected, is much slower.

Table 5: Timing experiments (in seconds) with $m=1.0 \mathrm{e}+04, n=1.0 \mathrm{e}+04, r=1.0 \mathrm{e}+01$.

\begin{tabular}{|c|c|c|c|c|c|c|c|c|}
\hline proj & ortho & StRSt_pj & RRR & ez-2nd & Shalit & KSL & KSL+ & geod \\
\hline $2.6 \mathrm{e}-02$ & $2.3 \mathrm{e}-02$ & $2.2 \mathrm{e}-02$ & $2.2 \mathrm{e}-02$ & $2.1 \mathrm{e}-02$ & $2.5 \mathrm{e}-02$ & $2.4 \mathrm{e}-02$ & $2.3 \mathrm{e}-02$ & $5.8 \mathrm{e}+01$ \\
\hline
\end{tabular}

To test how these figures may depend on the platform, we also ran the same experiments with Matlab 8.1.0.604 (R2013a) on a PC with four Intel(R) Xeon(R) CPU X3210 2.13GHz, $4096 \mathrm{~KB}$ cache each, running Linux kernel 2.6.18. The outcomes are presented in Tables 7 and (8).

\section{Retractions at work: low-rank matrix completion}

Several modern solvers for optimization on manifolds require the manifold of interest to be equipped with a retraction; see, in particular, the Manopt toolbox [BMAS14]. The behavior of the resulting optimization algorithm may crucially depend on the choice of the retraction. In this section, we illustrate this claim for the low-rank manifold $\mathcal{M}_{r}$ (2), by showing that the retractions discussed above yield considerable variations in performance when they are used within a Riemannian trust-region solver applied to a low-rank matrix completion problem. 
Table 6: Timing experiments (in seconds) with $m=1.0 \mathrm{e}+03, n=1.0 \mathrm{e}+04, r=1.0 \mathrm{e}+01$.

\begin{tabular}{|c|c|c|c|c|c|c|c|c|}
\hline proj & ortho & StRSt_pj & RRR & ez-2nd & Shalit & KSL & KSL+ & geod \\
\hline $1.5 \mathrm{e}-02$ & $1.3 \mathrm{e}-02$ & $1.3 \mathrm{e}-02$ & $1.3 \mathrm{e}-02$ & $1.3 \mathrm{e}-02$ & $1.5 \mathrm{e}-02$ & $1.5 \mathrm{e}-02$ & $1.3 \mathrm{e}-02$ & $3.6 \mathrm{e}+01$ \\
\hline
\end{tabular}

Table 7: Timing experiments (in seconds) with $m=1.0 \mathrm{e}+04, n=1.0 \mathrm{e}+04, r=1.0 \mathrm{e}+01$.

\begin{tabular}{|c|c|c|c|c|c|c|c|c|}
\hline proj & ortho & StRSt_pj & RRR & ez-2nd & Shalit & KSL & KSL+ & geod \\
\hline $1.3 \mathrm{e}-02$ & $9.9 \mathrm{e}-03$ & $1.1 \mathrm{e}-02$ & $1.0 \mathrm{e}-02$ & $1.1 \mathrm{e}-02$ & $1.2 \mathrm{e}-02$ & $1.2 \mathrm{e}-02$ & $1.1 \mathrm{e}-02$ & $2.5 \mathrm{e}+01$ \\
\hline
\end{tabular}

The objective function $f$ on $\mathcal{M}_{r}$ is the one described in AAM14, namely,

$$
f(X)=\frac{1}{2}\left\|\mathcal{P}_{\Omega}(X-C)\right\|_{F}^{2},
$$

where $C$ is an $m \times n$ matrix, $\Omega \subset\{1, \ldots, m\} \times\{1, \ldots, n\}$ is the set of indices of the observed entries, $\mathcal{P}_{\Omega}$ sets to zero the entries not in $\Omega$ while leaving the other entries unchanged, and $\|\cdot\|_{F}$ denotes the Frobenius norm. A minimizer of $f$ over $\mathcal{M}_{r}$ is thus a rank-r matrix $X$ such that $X_{i j}-C_{i j},(i, j) \in \Omega$, is smallest in the least-square sense.

We conducted several numerical experiments with Matlab R2014a equipped with the Manopt toolbox, version 1.0.7. We chose the manifold structure of $\mathcal{M}_{r}$ to be the one generated by fixedrankembeddedfactory (the geometry described in Van13]), with the exception of the retraction which was reassigned in turn to each of the retractions defined in Section 3 . We experimented with various solvers (steepestdescent, conjugategradient, and trustregions), various procedures for constructing $C$, and various procedures for constructing the initial guess $X_{0}$ passed to the solver. We refer to this combination of choices as the setup.

The outcomes of the numerical experiments were very diverse and it is impractical to describe them in detail in view of the large number of setups that were considered. The big picture is that, for some setups, the choice of the retraction has little impact on the behavior of the algorithm, while for other setups, the choice of the retraction has a significant impact, with qualitative findings that may or may not be consistent across runs. (Runs differ when the procedures for constructing $C$ or $X_{0}$ involve (pseudo)randomly generated matrices.)

In Table 9, we report results obtained with a setup for which the qualitative findings were fairly consistent across runs. Matrix $C$ is generated as $C=L R^{\top}$, where $L$ of size $m \times r$ and $R$ of size $n \times r$ are drawn from the standard normal distribution. The index set $\Omega$ is chosen uniformly at random with a sampling ratio of $4 d /(m n)$, where $d=k(m+n-k)$ is the dimension of $\mathcal{M}_{r}$. The initial iterate $X_{0}$ is chosen as the best rank-r approximation of $\mathcal{P}_{\Omega}(C)$ (obtained with an SVD of $\mathcal{P}_{\Omega}(C)$ truncated to its $r$ largest singular values). Manopt's factory fixedrankembeddedfactory and solver trustregions are called with default parameters. The number of trust-region inner iterations in Table 9 correspond to one typical run 1 In this specific setup, we found across all runs that retractions proj, ortho, KSL, and geod perform similarly, and better than the other retractions. Moreover, the ratio between the number of inner iterations taken by the worst and the

\footnotetext{
${ }^{1}$ The Matlab code that we used to generate the tables of this paper is available from http://sites.uclouvain.be/absil/2013.04
} 
Table 8: Timing experiments (in seconds) with $m=1.0 \mathrm{e}+03, n=1.0 \mathrm{e}+04, r=1.0 \mathrm{e}+01$.

\begin{tabular}{|c|c|c|c|c|c|c|c|c|}
\hline proj & ortho & StRSt_pj & RRR & ez-2nd & Shalit & KSL & KSL+ & geod \\
\hline $6.9 \mathrm{e}-03$ & $5.8 \mathrm{e}-03$ & $5.9 \mathrm{e}-03$ & $5.8 \mathrm{e}-03$ & $5.8 \mathrm{e}-03$ & $6.8 \mathrm{e}-03$ & $6.5 \mathrm{e}-03$ & $6.0 \mathrm{e}-03$ & $1.5 \mathrm{e}+01$ \\
\hline
\end{tabular}

best retraction was most often slightly smaller than 2 . This shows that the choice of the retraction can have a considerable impact on the performance of low-rank optimization algorithms.

Table 9: Total number of inner iterations required to reach the first iterate number $k$ such that $f\left(X_{k}\right)=1.0 \mathrm{e}-06 f\left(X_{0}\right)$, with $m=1.0 \mathrm{e}+03, n=1.0 \mathrm{e}+03, r=1.0 \mathrm{e}+01$.

\begin{tabular}{|c|c|c|c|c|c|c|c|c|c|}
\hline & proj & ortho & StRSt-pj & RRR & ez-2nd & Shalit & KSL & KSL+ & geod \\
\hline \# iter & 16 & 19 & 29 & 25 & 20 & 27 & 16 & 27 & 16 \\
\hline
\end{tabular}

\section{Concluding comments}

We have presented, analyzed, and tested numerically several retractions on the low-rank manifold $\mathcal{M}_{r}$ of rank- $r m \times n$ matrices. In the absence of a closed-form expression, the exponential retraction (geod) is clearly the least time-efficient one, confirming that much computational effort can be spared by considering other retractions. A rather good coherence has been observed between flop counts and run times, but the differences between the various retractions along these criteria are rather inconsequential, except for geod. However, the various retractions differ markedly according to properties such as domain of definition, boundedness, first/second-order property, and symmetry. It also appears from the various low-rank matrix completion experiments mentioned in Section 5 that the choice of the low-rank retraction is an issue that deserves attention when a practical low-rank optimization problem is solved: in the seemingly innocuous setup used for Table 9, replacing a retraction by an other can make the algorithm twice faster.

It is quite possible that one of the low-rank retractions will emerge from extended practice as the most adequate default choice within standard solvers, but it is premature to venture a definitive recommendation. The current state of affairs is that the developers of low-rank numerical methods have now available a sizeable collection of mechanisms - the various retractions presented in Section 3 - to smoothly produce curves of fixed-rank matrices with given initial position and velocity; and we know from Table 9 that the choice of the mechanism may have a noticeable impact on the performance of the numerical method.

\section{Acknowledgements}

We are grateful to the anonymous referees and to Bart Vandereycken for several useful comments on the first version of this paper. 


\section{References}

[AAM14] P.-A. Absil, Luca Amodei, and Gilles Meyer. Two Newton methods on the manifold of fixed-rank matrices endowed with riemannian quotient geometries. Computation. Stat., 29(3-4):569-590, 2014. doi:10.1007/s00180-013-0441-6.

[ABG07] P.-A. Absil, C. G. Baker, and K. A. Gallivan. Trust-region methods on Riemannian manifolds. Found. Comput. Math., 7(3):303-330, July 2007. doi:10.1007/s10208-005-0179-9

$\left[\mathrm{ADM}^{+} 02\right]$ Roy L. Adler, Jean-Pierre Dedieu, Joseph Y. Margulies, Marco Martens, and Mike Shub. Newton's method on Riemannian manifolds and a geometric model for the human spine. IMA J. Numer. Anal., 22(3):359-390, July 2002. doi:10.1093/imanum/22.3.359

[AM12] P.-A. Absil and Jérôme Malick. Projection-like retractions on matrix manifolds. SIAM J. Optim., 22(1):135-158, 2012. doi:10.1137/100802529.

[AMS08] P.-A. Absil, R. Mahony, and R. Sepulchre. Optimization Algorithms on Matrix Manifolds. Princeton University Press, Princeton, NJ, 2008. URL: http://press.princeton.edu/titles/8586.html.

[BMAS14] Nicolas Boumal, Bamdev Mishra, P.-A. Absil, and Rodolphe Sepulchre. Manopt, a matlab toolbox for optimization on manifolds. Journal of Machine Learning Research, 15:1455-1459, 2014. URL: http://jmlr.org/papers/v15/boumal14a.html.

[Boo03] William M. Boothby. An Introduction to Differentiable Manifolds and Riemannian Geometry. Academic Press, 2003. Revised Second Edition.

[GV96] Gene H. Golub and Charles F. Van Loan. Matrix Computations. Johns Hopkins Studies in the Mathematical Sciences. Johns Hopkins University Press, Baltimore, MD, third edition, 1996.

[KL07] O. Koch and C. Lubich. Dynamical low-rank approximation. SIAM Journal on Matrix Analysis and Applications, 29(2):434-454, 2007. doi:10.1137/050639703.

[KSV14] Daniel Kressner, Michael Steinlechner, and Bart Vandereycken. Low-rank tensor completion by Riemannian optimization. BIT Numerical Mathematics, 54(2):447468, 2014. doi:10.1007/s10543-013-0455-z.

[Lee03] John M. Lee. Introduction to smooth manifolds, volume 218 of Graduate Texts in Mathematics. Springer-Verlag, New York, 2003.

[LO13] Ch. Lubich and I.V. Oseledets. A projector-splitting integrator for dynamical lowrank approximation, 2013. arXiv:1301.1058v2,

[Lue72] David G. Luenberger. The gradient projection method along geodesics. Management Sci., 18:620-631, 1972. 
[MMBS13a] B. Mishra, G. Meyer, F. Bach, and R. Sepulchre. Low-rank optimization with trace norm penalty. SIAM J. Optim., 23(4):2124-2149, 2013. doi:10.1137/110859646.

[MMBS13b] B. Mishra, G. Meyer, S. Bonnabel, and R. Sepulchre. Fixed-rank matrix factorizations and Riemannian low-rank optimization, 2013. arXiv:1209.0430v2,

[MS14] B. Mishra and R. Sepulchre. R3MC: A Riemannian three-factor algorithm for lowrank matrix completion, 2014. Accepted for publication in the proceedings of the 53rd IEEE Conference on Decision and Control, 2014. arXiv:1306.2672v2.

[Ros61] J. B. Rosen. The gradient projection method for nonlinear programming. II. Nonlinear constraints. J. Soc. Indust. Appl. Math., 9:514-532, 1961.

[RW12] Wolfgang Ring and Benedikt Wirth. Optimization methods on Riemannian manifolds and their application to shape space. SIAM J. Optim., 22(2):596-627, 2012. doi:10.1137/11082885X

[Shu86] Michael Shub. Some remarks on dynamical systems and numerical analysis. In L. Lara-Carrero and J. Lewowicz, editors, Proc. VII ELAM., pages 69-92. Equinoccio, U. Simón Bolívar, Caracas, 1986.

[SWC13] Uri Shalit, Daphna Weinshall, and Gal Chechik. Online learning in the embedded manifold of low-rank matrices. J. of Mach. Learn. Res., 13:429-458, 2013.

[Van13] Bart Vandereycken. Low-rank matrix completion by Riemannian optimization. SIAM J. Optim., 23(2):1214-1236, 2013. doi:10.1137/110845768. 